\title{
Absence of Adenylyl Cyclase 3 Perturbs Peripheral Olfactory Projections in Mice
}

\author{
Dong-Jing Zou, Alexander T. Chesler, Claire E. Le Pichon, Andriy Kuznetsov, Xin Pei, Eugene L. Hwang, and \\ Stuart Firestein \\ Department of Biological Sciences, Columbia University, New York, New York 10027
}

\begin{abstract}
A remarkable feature of peripheral olfactory projections in mammals is the convergence of axons from olfactory sensory neurons (OSNs) expressing the same odorant receptor (OR) into the same glomeruli. There is mounting evidence that the ORs play critical roles in glomerular formation. However, it remains unclear how the OR exerts its function of sorting axons into homogeneity. We and others have shown previously that activation of the G-protein/cAMP signaling cascade underlies glomerular formation. Here, we further investigated whether establishment of the mature glomerular array requires adenylyl cyclase 3 (AC3), a key component of the OR-mediated cAMPdependent signaling cascade. We found robust AC3 expression in both OSN cilia and axons during the period of active glomerular formation in neonatal mice. Examination of OR-tagged mice in an AC3 knock-out background revealed that the absence of AC3 drastically and differentially perturbed the formation of several representative glomeruli. Furthermore, heterogeneous glomeruli innervated by axons of multiple OSN populations persisted in such mice well into adulthood. In addition, reproducible aberrations in axonal projections in $\mathrm{AC} 3-1-$ mice appeared to correlate with the activation of specific OR loci, regardless of the expressed receptor sequence, suggesting that $\mathrm{OR}$ expression is but one factor in determining OSN axonal projections. Together, our results indicate that cAMP signaling is critical for axonal sorting and the establishment of axonal identity.
\end{abstract}

Key words: glomerulus; activity-dependent; development; signal transduction; transgenic mice; plasticity

\section{Introduction}

Accurate sensory perception relies on precise connections between peripheral sensory neurons and the brain. In the olfactory system, this is achieved by organizing the projections of axons from olfactory sensory neurons (OSNs) according to the odorant receptor (OR) they express (Mombaerts et al., 1996). In mice, each OSN expresses only one of $\sim 1100$ ORs (Buck and Axel, 1991; Zhang and Firestein, 2002). Although OSNs expressing the same receptor are dispersed throughout the olfactory epithelium (Ressler et al., 1993; Vassar et al., 1993), their axons coalesce to form a structure, called a glomerulus, in the olfactory bulb (Mombaerts et al., 1996; Treloar et al., 2002).

In other sensory systems, patterned electrical activity is thought to refine coarse projections initially established through activity-independent processes (Katz and Shatz, 1996; Grubb and Thompson, 2004). The role of activity in the formation of olfactory projections, however, is less clearly defined. Several experiments in which various components of the odor-evoked signal

Received Feb. 15, 2007; revised April 16, 2007; accepted May 14, 2007.

This work was supported by grants from the National Institutes of Health, National Institute on Deafness and Other Communication Disorders (S.F.). We thank Dr. Daniel Storm of the University of Washington (Seattle, WA) for the AC3 heterozygous mice, and Drs. Paul Feinstein, Anne Vassalli, and Peter Mombaerts of the Rockefeller University (New York, NY) for the OR gene-targeted mice. We are grateful to Zita Peterlin and Jessica Brann for comments on this manuscript. We appreciate Dr. Helen Treloar of Yale University (New Haven, CT) for sharing her unpublished data on the expression patterns of $\mathrm{AC} 3$ in the olfactory bulb.

Correspondence should be addressed to either Dong-Jing Zou or Stuart Firestein, 1212 Amsterdam Avenue, New York, NY 10027. E-mail: dz98@columbia.edu or sjf24@columbia.edu.

DOI:10.1523/JNEUROSCI.0699-07.2007

Copyright $\odot 2007$ Society for Neuroscience $\quad$ 0270-6474/07/276675-09\$15.00/0 cascade have been knocked out exhibited little alteration in glomerular formation. The gross organization of glomeruli in mice lacking cyclic nucleotide-gated channel A2 subunit, a crucial subunit of the cyclic-nucleotide-gated channel, appears mostly normal (Baker et al., 1999; Lin et al., 2000), except for the presence of supranumerary glomeruli innervated by OSNs expressing the OR M72 (Zheng et al., 2000). Normal glomerular formation is also reported in newborn mice lacking the key G-protein subunit G $\alpha$ olf (Belluscio et al., 1998). These observations led to the notion that glomerular development might be primarily directed by genetic factors.

However, previous data has demonstrated that activity is indeed required for proper establishment and maintenance of glomeruli. Sensory deprivation by naris closure perturbs glomerular maturation (Zou et al., 2004), whereas odor stimulation accelerates it (Kerr and Belluscio, 2006). Similarly, reduction of electrical excitability and inhibition of neural transmitter release in the OSNs can influence the establishment and maintenance of glomeruli (Yu et al., 2004).

Regardless of any controversy on the roles of activity, the OR itself clearly participates in glomerular formation (Mombaerts et al., 1996; Wang et al., 1998; Feinstein and Mombaerts, 2004; Feinstein et al., 2004). Previously, we and others have shown that OR activation of the G-protein/cAMP signaling cascade underlies glomerular formation (Imai et al., 2006; Chesler et al., 2007). Furthermore, glomerular structure is highly aberrant in mice deficient for adenylyl cyclase 3 (AC3), a critical component in the odor-evoked cAMP-dependent signaling pathways (Trinh and 
Storm, 2003). Although the AC3-/- mice represent the only knock-out of a key olfactory signaling cascade component to severely disrupt glomerular structure, the formation of ORspecific glomeruli has not been analyzed in detail in this genetic background.

Here, we show that the formation of OR-specific glomeruli is drastically and differentially perturbed in the AC3-/- background. Furthermore, under these conditions, reproducible perturbation of axonal projections is observed independent of the receptor expressed. Thus, the OR is but one factor important for glomerular formation. Our results suggest that proper development of OSN projections requires the activation of cAMPdependent signaling pathways.

\section{Materials and Methods}

Animals. AC3 heterozygous $(\mathrm{AC} 3+/-)$ mice were provided by Dr. Daniel Storm of the University of Washington (Seattle, WA) (Wong et al., 2000). OR-tagged mice M71-IRES-tauGFP (taulacZ), M72-IREStauGFP (taulacZ), MOR23-IRES-tauGFP (taulacZ), and P2-IREStaulacZ, as well as receptor-replacement mice M71 $\rightarrow$ P2-IRES-taulacZ and MOR23 $\rightarrow$ M71-IRES-taulacZ were provided by Drs. Paul Feinstein, Anne Vassalli, and Peter Mombaerts of the Rockefeller University (New York, NY) (Vassalli et al., 2002; Feinstein and Mombaerts, 2004; Feinstein et al., 2004). In these OR gene-targeted mice, a sequence encoding the internal ribosome entry site (IRES) and a marker (either tauGFP or taulac $Z$ ) is inserted downstream of a given receptor coding region (herein referred to as OR-GFP or OR-lacZ mice). With this strategy, all of the OSNs expressing the same receptor and their axons are marked with the cotranslated green fluorescent protein (GFP) or $\beta$-galactosidase ( $\beta$ gal) encoded by the bacterial gene lac $Z$. Therefore, in these mice, the convergence of labeled OSN axons (herein referred to as OR axons) into glomeruli (herein referred to as OR glomeruli) is readily visible either in whole-mount or in serial coronal sections through the olfactory bulb.

The OR gene-targeted homozygous mice were first crossed with $\mathrm{AC} 3+/-$ mice. Their offspring in the AC3 $+/-$ background were crossed again. Among the next generations, those in the AC $3+/+$ and AC3 $-/-$ background were used for data analysis, whereas those in the AC $3+/-$ background that were also homozygous for the tagged ORs were used for additional breeding. To increase the survival of AC3-/mice, litters were trimmed to $3-5$ pups per litter within the first $24 \mathrm{~h}$ after birth.

All gene-targeted animals were in a mixed $129 \times$ B6 background and were housed with a $12 \mathrm{~h}$ light/dark cycle in cages with a microfilter lid. Experiments were performed according to protocols approved by the Columbia University Institutional Animal Care and Usage Committee.

In situ hybridization. In situ hybridization was performed on $12 \mu \mathrm{m}$ coronal sections through the olfactory epithelium and olfactory bulb as described previously (Ishii et al., 2004). The olfactory marker protein (OMP) probe was designed to the coding region and cloned from olfactory epithelium cDNA using primers 5'TCCGAGTAGAGAGCCTGAAGC3' and 5'CCACAGAGGCCTTTAGGTTG3'. The probe for AC3 was generated from a full-length cDNA clone (I.M.A.G.E. ID 6835448) obtained from Open Biosystems (Huntsville, AL).

Images of the sections subjected to chromogenic in situ hybridization were taken with a SPOT digital camera (Diagnostic Instruments, Sterling Heights, MI). For those sections subjected to two-color in situ hybridization, a single optical section was obtained with a confocal microscope (FluoView 600; Olympus, Tokyo, Japan).

Immunohistochemistry. Immunohistochemistry was performed as previously described (Zou et al., 2004) in serial coronal sections $(16 \mu \mathrm{m})$ through the olfactory epithelium and the olfactory bulb of homozygous OR-lacZ (or GFP) mice in either the AC3 + / + or AC3 - / - background. The primary antibodies used were rabbit anti-AC3 (1:500; Santa Cruz Biotechnology, Santa Cruz, CA), goat anti-OMP (1:1500; Wako, Richmond, VA), rabbit anti- $\beta$-galactosidase (1:1000; Cappel, Cochranville, PA), rabbit anti-GFP (1:500; Invitrogen, Eugene, OR), and goat antiolfactory cell adhesion molecule (OCAM; 1:1000; R \& D Systems, Min- neapolis, MN). The secondary antibodies were Alexa Fluor 488conjugated donkey anti-rabbit (1:750; Invitrogen), and Alexa Fluor 594conjugated donkey anti-goat (1:750; Invitrogen). Nucleic dye TOTO3 (Invitrogen) was used at 1:10,000.

Western blot assay. Total protein from the olfactory epithelium and the olfactory bulb of postnatal day 0 (PD0) mice was extracted in a lysis buffer containing $1 \times \mathrm{PBS}, \mathrm{pH} 7.4,1 \% \mathrm{NP}-40,0.5 \%$ sodium deoxycholate, and $1 \times$ Complete Mini Protease Inhibitors mix (Roche, Indianapolis, IN). The protein concentration of the supernatant was measured using a Coomassie protein assay kit (Pierce, Rockford, IL). Denatured samples $(50 \mu \mathrm{g})$ were separated on $8 \%$ acrylamide gels by SDS-PAGE. After transfer to polyvinylidene difluoride membrane, the blots were blocked for $1 \mathrm{~h}$ at room temperature (RT) with $3 \%$ nonfat dry milk in the TBS buffer containing $0.1 \%$ Tween 20 . They were then incubated with $200 \mathrm{ng} / \mathrm{ml}$ rabbit anti-AC3 antibody (Santa Cruz) or $500 \mathrm{ng} / \mathrm{ml} \mathrm{rabbit}$ anti-actin antibody (Novus Biologicals, Littleton, CO) overnight at $4^{\circ} \mathrm{C}$, followed by an incubation with horseradish peroxidase (HRP)conjugated donkey anti-rabbit IgG for $1 \mathrm{~h}$ at RT. Blots were visualized by Western chemiluminescent HRP substrate (Millipore, Billerica, MA) as per the manufacturer's instructions.

Imaging acquisition. For the whole-mount X-gal staining of ORspecific projections, anesthetized OR-lacZ mice were perfused with $4 \%$ paraformaldehyde (PFA) in $0.1 \mathrm{M}$ phosphate buffer (PB), pH7.4, for 5 min. Olfactory bulbs were dissected and incubated in a solution containing $5 \mathrm{~mm}$ potassium-ferricyanide, $5 \mathrm{~mm}$ potassium-ferrocyanide, and 1 $\mathrm{mg} / \mathrm{ml} \mathrm{X}$-gal in PBS at $37^{\circ} \mathrm{C}$ overnight. To observe the projections of GFP+ OSN axons in whole mount, animals were perfused briefly with $4 \%$ PFA in $0.1 \mathrm{M} \mathrm{PB}$ for $1 \mathrm{~min}$ and olfactory bulbs were dissected. Images of the whole-mount bulbs were taken with a SPOT digital camera or a confocal microscope (Olympus FluoView 600).

Optical sections $(2 \mu \mathrm{m})$ through the depth of the immunostained coronal sections or the whole-mount bulb were taken with a confocal microscope (Olympus FluoView 600) and analyzed with the ImageJ, NIH Image, and Adobe (San Jose, CA) Photoshop programs. Images of Z-series optical sections projected to a single plane were not modified other than to balance brightness and contrast.

\section{Results}

\section{Axonal expression of AC3 in neonatal mice}

Previous work has demonstrated that in adult mice AC3 is highly concentrated in the cilia of mature OSNs, where it serves as a critical component of the odor-evoked signaling cascade (Bakalyar and Reed, 1990). Here, we investigated AC3 expression patterns in the olfactory epithelium and olfactory bulb of neonatal mice (PD0-PD5) by in situ hybridization (Fig. 1) and by immunohistochemistry (Fig. 2). In newborn mice (PD0), chromogenic in situ hybridization showed that AC3 was highly expressed in the epithelium, but was not detected in the bulb (Fig. 1A). At PD5, the intensity of the AC3 signal had increased in the epithelium without appreciable signals in the bulb (Fig. $1 B$ ). The neonatal AC3 expression in the bulb thus differs from that reported in adults (Visel et al., 2006).

To characterize which cell types express AC3 in the epithelium of neonatal mice, we used two-color in situ hybridization to detect both AC3 and OMP, a marker of mature OSNs. At PD0, intense AC3 staining overlapped with that of OMP in many OSNs (Fig. 1C). However, low levels of AC3 expression were also observed in the immature, OMP-negative layer of the epithelium (Fig. 1C, bottom). By PD5, the AC3 mRNA signals had become more intense and were now predominantly restricted to the $\mathrm{OMP}+$ mature OSNs (Fig. 1D). The constraint of AC3 message to mature cells is similar to that seen in adult mice.

However, the pattern of AC3 protein expression in neonatal mice differed from the localization typically observed in adult tissues. We found that at PD0 AC3 was not only expressed in the OSN cilia, but also in the bulb; strong AC3 immunoreactivity was 


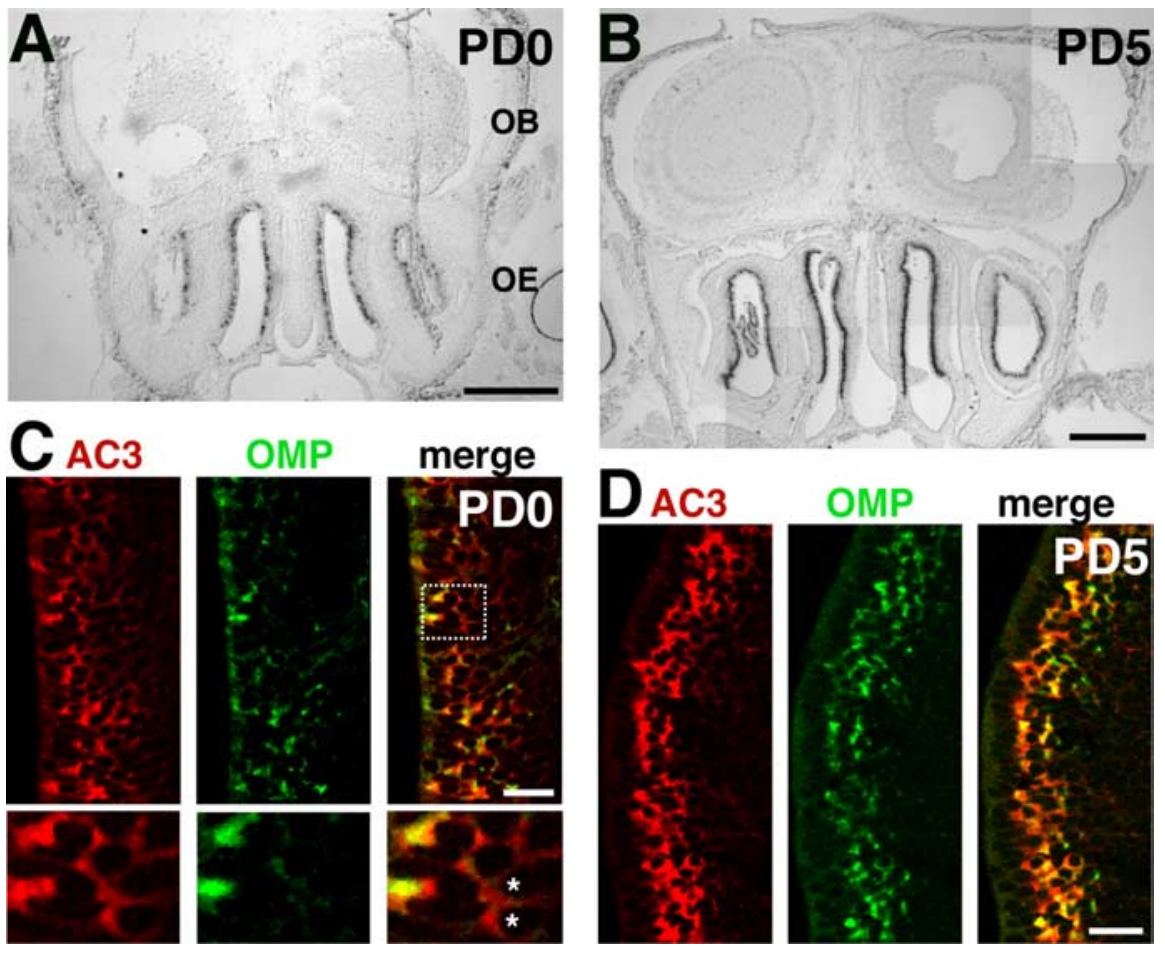

Figure 1. $A C 3$ mRNA expression in the olfactory epithelium and the olfactory bulb during early postnatal development. $A, B$ $A C 3$ transcripts detected by chromogenic in situ hybridization in the olfactory epithelium (OE) and the olfactory bulb (OB) at PDO $(\boldsymbol{A})$ and PD5 (B). C, D, Distribution of AC3 transcripts detected by two color in situ hybridization in the epithelium on the septum at PD0 (C) and PD5 (D). Green, 0MP; red, AC3. The bottom of $\boldsymbol{C}$ (enlarged view of the box in $\boldsymbol{C}$ ) shows that some AC3-positive cells are OMP negative (asterisk) at PD0. D, By PD5, AC3 expression is restricted to the OMP-positive cells. Scale bars: $\boldsymbol{A}, \boldsymbol{B}, 500 \mu \mathrm{m} ; \boldsymbol{C}, \boldsymbol{D}, 25 \mu \mathrm{m}$.

detected in olfactory sensory axons in the olfactory nerve layer and in the glomerular layer (Fig. 2A). As a control, AC3 immunoreactivity was completely absent from AC3-/- mice (Fig. $2 B$ ) (Wong et al., 2000). Although at PD5 AC3 protein expression in the olfactory nerve layer was diminished relative to PD0, AC3 expression was still readily detectable in individual glomeruli (Fig. 2C). We noted no AC3 protein expression in the somata of mitral cells. The presence of AC3 protein in the bulb at PD0 was further confirmed by Western blot (Fig. 2D).

\section{Abnormal glomerular structures in AC3 knock-out mice}

The unexpected localization of AC3 protein in OSN axons was most apparent between PD0 and PD5, a period of robust sorting of OSN axons into glomeruli. This result raised the possibility that, in addition to its role in odor-evoked signal transduction in the epithelium, AC3 also plays an important role in glomerular development. Indeed, in the AC3 knock-out mice the gross structure of glomeruli is highly abnormal (Trinh and Storm, 2003). Instead of the densely packed discrete glomeruli typically seen in the bulb of wild-type mice at PD20 (Fig. 2E), glomeruli in agematched $\mathrm{AC} 3-/-$ mice appeared to be fewer in number and larger in size in the dorsal bulb. Also, in other regions, the boundaries between neighboring glomeruli were at times poorly defined (Fig. $2 F$ ). Although the gross structure appears abnormal, both the dendritic marker microtubule associated protein 2 and the synaptic marker synaptophysin could be detected by immunohistochemistry in the glomerular layer of AC3-/- mice (data not shown), indicating that at least some of the OSN axons made synapses with the dendrites of bulbar neurons despite the absence of AC3 expression. Termination of OSN axons in the appropriate bulbar layer was another feature perturbed by the lack of AC3 expression. By $\mathrm{PD} 20, \mathrm{OSN}$ axons in AC3+/+ mice were restricted to the glomerular layer. In contrast, in $\mathrm{AC} 3-/-$ mice there was widespread aberrant overshooting of OSN axons into the external plexiform layer, and even deep into the granule cell layer (Fig. 2G).

\section{Projection of $\mathrm{P} 2$ axons in the AC3-/- background}

To better elucidate the ramifications of AC3 deletion on the development of the olfactory bulb, we investigated the projections of individual OSN axon populations in this genetic background. We have chosen four OR-specific populations to examine (Vassalli et al., 2002; Feinstein and Mombaerts, 2004; Feinstein et al., 2004). These receptors are highly diverse in their sequence and expression patterns in the epithelium, as well as in the timing of their formation and the position of their glomeruli in the bulb. We anticipate that the formation of these distinctive OR-specific glomeruli may therefore be representative, to a large extent, of the steps required for the establishment of the entire glomerular array.

The glomeruli formed by axons of OSNs expressing the $\mathrm{P} 2$ receptor, the first OR allele to be marked by gene-targeting (Mombaerts et al., 1996), have been the subject of extensive studies on the mechanisms governing glomerular development. As a comparative study, we examined whether the lack of AC3 expression affects the development of P2 glomeruli. P2-expressing OSNs are located in the intermediate region along the dorsalventral axis of the epithelium [what is labeled as zone II by Ressler et al. (1993)] and their axons project to two glomeruli positioned symmetrically in the ventral bulb, one in the medial half-bulb and one in the lateral half-bulb. In P2-lacZ mice wild-type for AC3 (Fig. 3A), whole-mount X-gal staining at PD5 revealed numerous $\mathrm{P} 2$-expressing OSNs in the epithelium. In AC3-/- mice (Fig. $3 B$ ), the zonal position was preserved, but there were fewer P2expressing OSNs compared with those in AC3+/+ littermates.

In the $\mathrm{AC} 3+/+$ background, the convergence of $\mathrm{P} 2$ axons into glomeruli was readily observed in both the medial (Fig. $3 A^{\prime}$ ') and lateral half-bulb. In stark contrast, in the AC3-/- background, we failed to observe distinct P2 glomeruli in either the medial (Fig. 3B') or lateral half-bulb. Instead, only a small number of P2 axons projected diffusely over a relatively restricted region that corresponded to the general area of the normal P2 glomerulus. The perturbation of $\mathrm{P} 2$ axonal projections in AC3 $-/-$ mice persisted in older animals (Fig. $3 C-D$ ). Even at PD120, P2 axons remained largely dispersed within this same restricted region of the bulb (Fig. 3D). Thus, although the broad projection of the axons appeared normal, the deficiency in AC3 reduced the number of $\mathrm{P} 2$-expressing OSNs and perturbed the formation of $\mathrm{P} 2$ glomeruli.

Next, we examined in greater detail the innervation patterns of P2 axons by immunohistochemistry. On serial coronal sections of the bulb, P2 axons were labeled with antibodies specific 
for $\beta$-gal and were compared with the entirety of the mature OSN axonal projections labeled with antibodies for OMP. The nucleic dye TOTO3, which stained the nuclei of juxtaglomerular cells surrounding the glomerulus, was used to delineate the glomerular structure. To further reduce any uncertainty in defining a single glomerulus, we always examined at least three consecutive immunostained sections. In immature $\mathrm{AC} 3+/+$ mice at PD5, we observed P2 glomeruli with overlapping immunoreactivity for both $\beta$-gal and OMP (Fig. $3 E$ ), showing that the glomerulus was innervated entirely and homogeneously by $\mathrm{P} 2$ axons. In contrast, in the AC3-/- background, even at PD60 (when most glomeruli have matured to a homogeneous state in AC3+/+ mice), we were unable to detect any homogeneous P2 glomeruli ( $n=4$ bulbs, ranging from PD20 to PD60). Consistent with our wholemount observations, isolated $\beta$-gal $+\mathrm{P} 2$ axons were found in multiple glomeruli (Fig. $3 F)$, where they entered and branched among axons from OSNs expressing different ORs $(\beta$-gal-/OMP +$)$.

Projection of MOR23 axons in the AC3-/- background

To determine whether the gross disruption of $\mathrm{P} 2$ axon projections in the absence of AC3 could be generalized to other OSN populations, we examined the formation of MOR23 glomeruli. Unlike the ventrally located P2-expressing cells, OSNs expressing MOR23 are distributed in the most dorsal part of the epithelium (zone I). Furthermore, whereas P2 glomeruli begin to form before birth and are positioned in the medial ventral bulb (Royal and Key, 1999), MOR23 glomeruli are formed postnatally and are located in the anterior dorsal bulb (Vassalli et al., 2002).

In MOR23-lacZ mice with normal AC3 expression, a single MOR23 glomerulus was typically seen either in the medial (Fig. $4 A$ ) or lateral half-bulb by PD20. In contrast, MOR23 axons in AC3-/- mice projected to multiple locations in each of the half-bulbs at this same age (Fig. $4 B$ ). Even in older animals, the multiple pro-

jection sites of MOR23 axons remained broadly scattered in the anterior bulb [PD60 (Fig. 4C); PD90 (Fig. 4D)]. Interestingly, it appeared that at PD90 the number of MOR23-expressing OSNs observed in the AC3-/- background (Fig. $4 E$ ) was not dramatically different from that in the AC3+/+ background (Fig. 4F). This result was in contrast to the apparent reduction of P2expressing OSNs in AC3-/- mice (Fig. 3), indicating that the absence of AC3 differentially influences the number of OSNs in an OR specific manner.

Next we sought to determine whether the lack of AC3 expression influenced the innervation patterns of MOR23 axons by
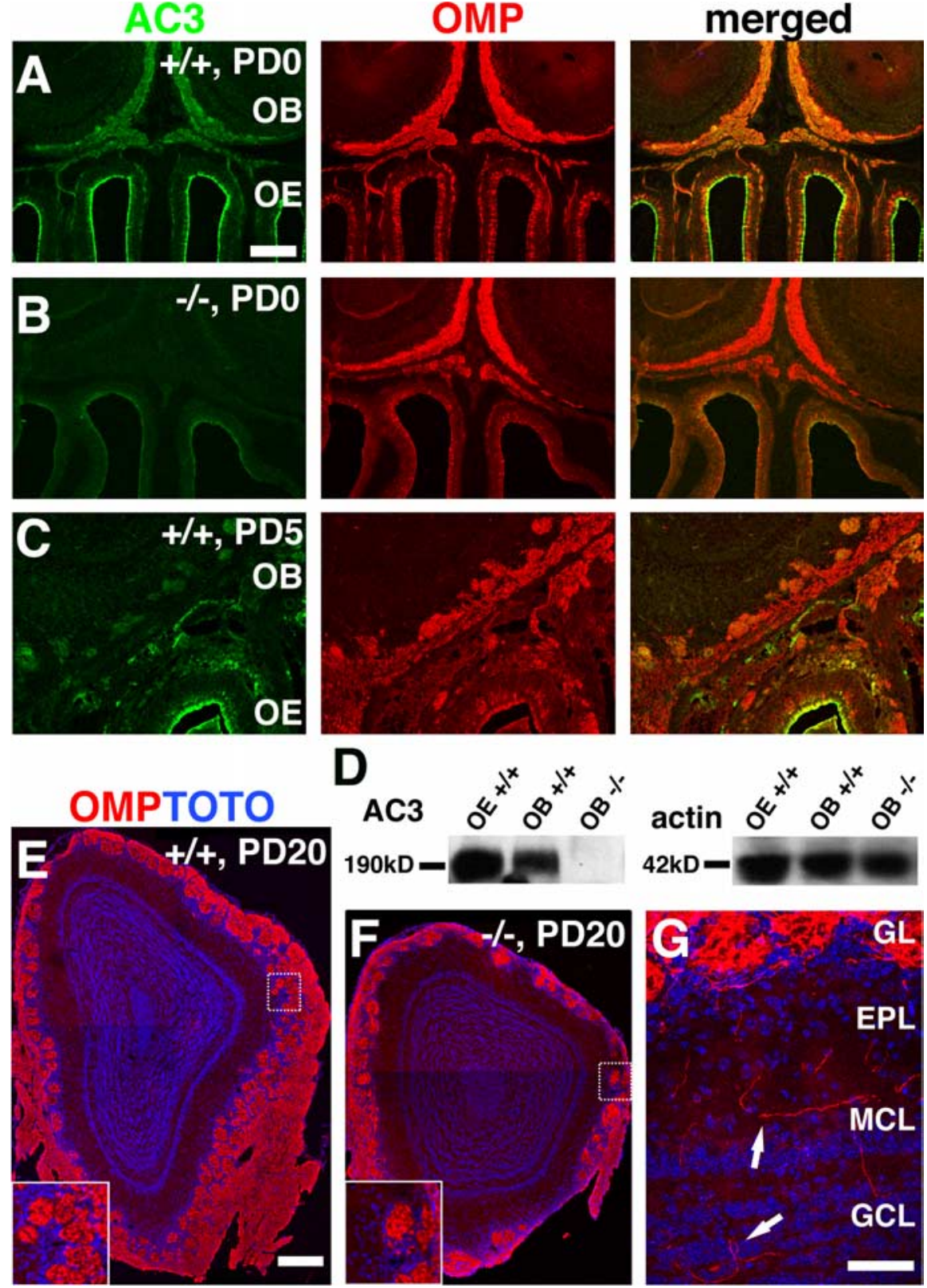

Figure 2. Expression of $\mathrm{AC} 3$ in olfactory axons and abnormal glomerular structures in $\mathrm{AC} 3-1-$ mice. $A, \mathrm{AC} 3$ immunoreactivity in the olfactory epithelium (OE) and the olfactory bulb (OB) at PDO. Green, $A C 3$; red, OMP. B, Absence of AC3 immunoreactivity in the epithelium and bulb in AC3 - / - mice at PD0. C, AC3 immunoreactivity in the epithelium and bulb at PD5. D, AC3 expression in the bulb at PDO detected by Western blot. Left, AC3 expression; right, actin expression showing the equal loading of total

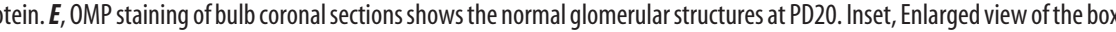
Red, OMP; blue, nucleic staining with TOT03 marks glomeruli. $F$, Abnormal glomeruli in AC3 - - - mice at PD20. Inset, Enlarged view of the box. G, Overshooting of OSN axons (arrows) in the bulb of AC3 - / - mice at PD20. GL, Glomerular layer; EPL, external plexiform layer; MCL, mitral cell layer; GCL, granule cell layer. Scale bars: (in $A) A-C, 200 \mu \mathrm{m}$; (in $E) E, F, 200 \mu \mathrm{m} ; \boldsymbol{G}, 50 \mu \mathrm{m}$.

immunohistochemistry. Whereas homogeneous MOR23 glomeruli were readily detected in the $\mathrm{AC} 3+/+$ background at PD10 (Fig. 4G), all MOR23 glomeruli in the AC3-/- background were heterogeneously innervated ( $n=5$ bulbs, ranging from PD20 to PD200). These heterogeneous glomeruli contained MOR23 axons $(\beta$-gal+/OMP +$)$ as well as axons from OSNs expressing other ORs ( $\beta$-gal-/OMP +$)($ Fig. $4 H)$. Such heterogeneous MOR23 glomeruli did not disappear even by PD200 (Fig. $4 I-J)$. Furthermore, we noted that in older animals multiple MOR23 glomeruli were readily observed in the same single coronal sections (Fig. 4I-K). This differed from younger animals in 

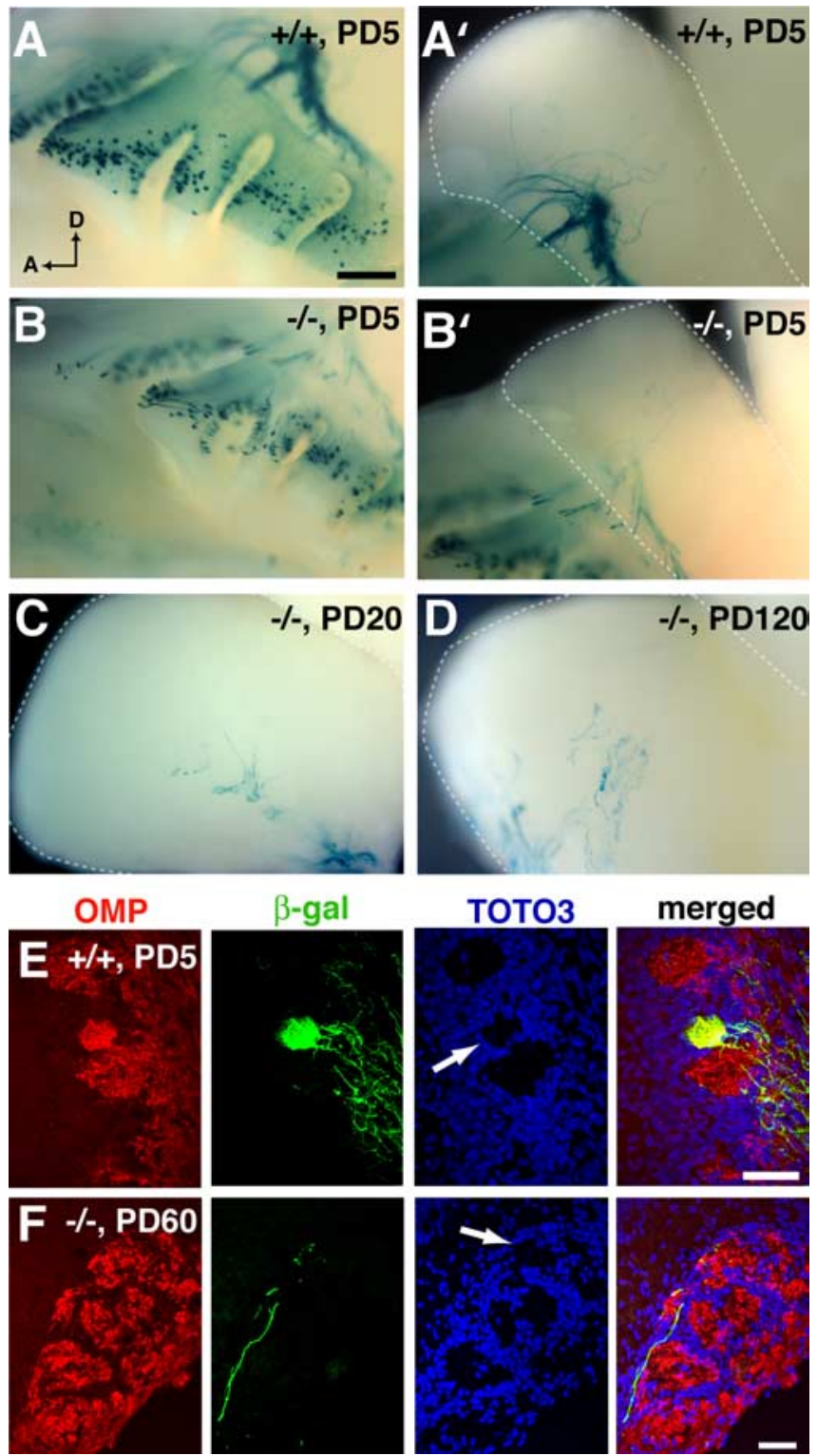

Figure 3. Deletion of $A C 3$ disrupts $P 2$ axonal projection. $A-D$, Medial view of $P 2$ axonal projections in whole-mount bulbs stained with X-gal. $A, P 2$-expressing 0 SNs in the epithelium of an $\mathrm{AC} 3+/+$ mouse at PD5 ( $\sim 200 \mathrm{X}$-gal-stained cells visible on the turbinates). A, Anterior; $D$, dorsal. $\boldsymbol{A}^{\prime}$, Convergence of $\mathrm{P} 2$ axons into a ventral glomerulus in the same mouse shown in $\boldsymbol{A}$. $B$, P2-expressing 0SNs in the epithelium of an AC3 - / - littermate at PD5 ( 125 X-galstained cells visible on the turbinates). $\boldsymbol{B}^{\prime}, \mathrm{A}$ few $\mathrm{P} 2$ axons are scattered in the bulb of the same $A C 3-1-$ mouse shown in $B$. C, D, P2 axons remained scattered in the bulbs of older $A C 3-/-$ mice ( $\boldsymbol{C}, \mathrm{PD} 20 ; \boldsymbol{D}, \mathrm{PD} 120) . \boldsymbol{E}, \boldsymbol{F}$, Organization of $\mathrm{P} 2$ axons by immunostaining of serial coronal sections through the bulb. Red, OMP staining labels mature OSN axons; green, $\beta$-gal staining labels P2-lacZ axons; blue, nucleic staining with TOT03 outlines individual glomeruli (arrow points to a glomerulus). $\boldsymbol{E}$, A homogeneous $\mathrm{P} 2$ glomerulus in the bulb of an $\mathrm{AC} 3+/+$ mouse at PD5. $\boldsymbol{F}, \mathrm{P2}$ axons detected by immunostaining of serial sections through the bulb of an $\mathrm{AC} 3-/-$ mouse at PD60. Scale bar: (in $\boldsymbol{A}) \boldsymbol{A}-\boldsymbol{D}, 0.5 \mathrm{~mm} ; \boldsymbol{E}, 50 \mu \mathrm{m} ; \boldsymbol{F}, 50 \mu \mathrm{m}$.

which we typically observed only a single MOR23 glomerulus in a single coronal section (Fig. $4 H$ ). It is possible that heterogeneous MOR23 glomeruli gradually accumulated in older animals because of the continual arrival of new axons.

Some MOR23 glomeruli were observed in more ventral/posterior regions in the bulb of AC3-/- mice. Such glomeruli remained negative for the OCAM (Yoshihara et al., 1997), a marker for ventrally projecting OSN axons and their glomeruli (Fig. $4 \mathrm{~K}$ ). We also noticed that there was an overall reduction of the
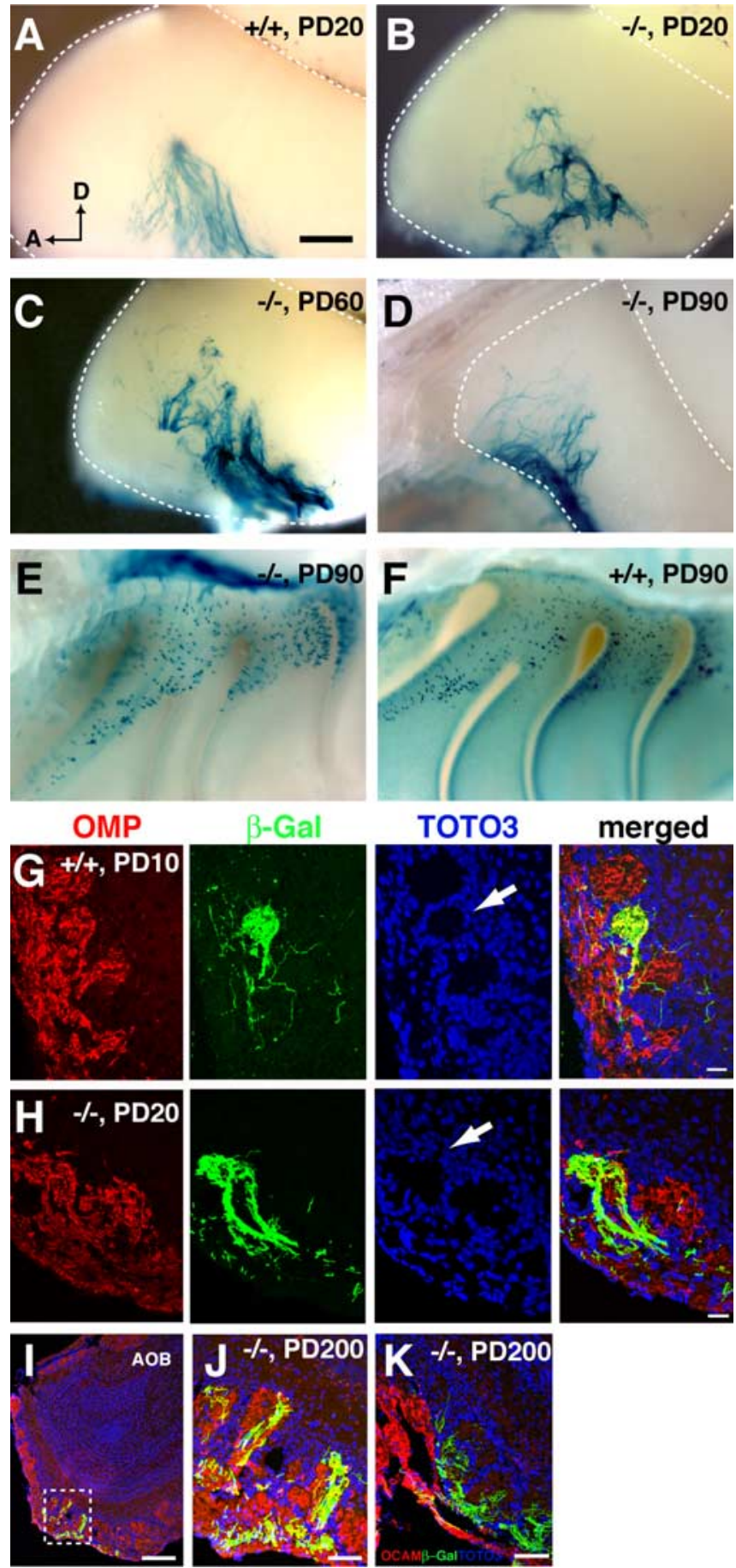

Figure 4. Deletion of AC3 disrupts MOR23 axonal projections. $A-D$, Medial view of MOR23 axonal projections in X-gal-stained whole mounts. $A$, Convergence of MOR23 axons into a single ventral glomerulus in a wild-type mouse at PD20. A, Anterior; D, dorsal. $\boldsymbol{B}-\boldsymbol{D}$, Diffuse MOR23 axonal projections in $\mathrm{AC3}-/-$ mice at PD20 (B), PD60 (C), and PD90 (D). E, MOR23-expressing OSNs in the epithelium ( $\sim 450$ cells visible on the turbinates) of the same $A C 3-/-$ mouse shown in $\boldsymbol{D}$ (PD90). $\boldsymbol{F}$, MOR23 neurons in the epithelium ( $\sim 450$ cells visible on the turbinates) of an $\mathrm{AC} 3+/+$ mouse at PD90. $\mathbf{G}-\boldsymbol{K}$, Organization of MOR23 axons detected by immun ostaining of serial coronal sections through the bulb. Red in G-J, OMP; in $\boldsymbol{K}, 0$ CAM, which labels OSN axons in the ventral bulb; green, $\beta$-gal. Blue, nucleic staining with T0T03 outlines individual glomeruli (arrow points to a glomerulus). G, A homogeneous MOR23 glomerulus in an $\mathrm{AC} 3+/+$ mouse at PD10. $\boldsymbol{H}, \mathrm{A}$ heterogeneous MOR23 glomerulus in the AC3-/- background at PD20. I, Multiple heterogeneous MOR23 glomeruli in AC3 - / - mice at PD200. J, Enlarged view of the box. $\boldsymbol{K}$, MOR23 glomeruli remained negative in the immunoreactivity for the OCAM. Scale bars: (in $\boldsymbol{A}) \boldsymbol{A}-\boldsymbol{F}, 0.5 \mathrm{~mm} ; \boldsymbol{G}, \boldsymbol{H}, 20 \mu \mathrm{m} ; \boldsymbol{I}, 200 \mu \mathrm{m} ; \boldsymbol{J}, 50 \mu \mathrm{m} ; \boldsymbol{K}, 50 \mu \mathrm{m}$. 
OCAM + region in the bulb of AC3-/mice. Together with the reduction in number of $\mathrm{P} 2$-expressing OSNs, the compression of the OCAM+ ventral bulb may reflect differential effects of AC3 elimination along the dorsal-ventral axis. Because of the gross differences in bulb structure between the $\mathrm{AC} 3+/+$ and $\mathrm{AC} 3-/-$ animals, the relative positions of MOR23 glomeruli in the two conditions could not be easily compared.

\section{Projections of M71 and M72 axons in the AC3-/- background}

P2 and MOR23 are very different receptors with low sequence similarity, different expression patterns in the epithelium, and different glomerular positions in the bulb. Their glomeruli also exhibit strikingly different defects in AC3-/- mice. But how does AC3 deficiency effect the axonal projections for OSN populations expressing two highly homologous receptors? M71 and M72 are ideal receptors to address this question as they have $96 \%$ identity, are both expressed in the dorsal zone of epithelium (zone I) and project to neighboring glomeruli in the posterior dorsal bulb.

In the AC3+/+ background, M71 axons reliably converged into glomeruli in the posterior dorsal bulbs (Fig. 5A). Strikingly, in AC3-/- littermates, M71 axons projected not only to the predicted posterior regions, but also to novel sites in the anterior dorsal bulb (Fig. 5B). Compared with the pronounced convergence of M71 axons in the AC3+/+ background (Fig. $5 C)$, projections of M71 axons were poorly organized in the AC3-/- background. Perturbation of axonal convergence was found in both the anticipated locations in the posterior bulb (Fig. 5D), as well as in the novel, atypical regions in the anterior bulb (Fig. 5E). Furthermore, the abnormal projections of M71 axons in the AC3-/ - background continued to be observed even in mice as old as PD200

(Fig. $5 F-G$ ) and, thus, this aberration is not merely a transient developmental event. Importantly, the novel anterior projections of M71 axons were consistently observed in all 12 AC3 - / - mice we examined (ranging from PD5 to PD200), suggesting that the absence of AC3 systematically disrupts the proper projection of M71 axons.

As we had done with the other receptor populations, we further examined the innervation patterns of M71 axons by immunohistochemistry. In contrast to the homogeneous M71 glomeruli seen in the AC3+/+ background (Zou et al., 2004), all M71 glomeruli observed in the AC3-/- background were heterogeneous ( $n=6$ bulbs, ranging from PD10 to PD200). The prevalence of heterogeneous glomeruli was found in both the expected and atypical projection sites (Fig. $5 H-I$ ). Consistent with the results from P2 and MOR23 glomeruli, heterogeneous M71 glomeruli persisted in older mice (PD200) (Fig. 5J).

In the AC3-/- background, the highly homologous receptor M72 demonstrated a phenotype of perturbations in axonal projections very similar to that of M71. In AC3-/- mice, M72 axons also formed atypical aberrant aggregations in the anterior dorsal bulb in addition to the expected posterior projections in the dorsal bulb (Fig. 6A). Likewise, immunostaining of serial coronal sections revealed that M72 glomeruli remained heterogeneous even after one year in both the anterior (Fig. $6 B$ ) and the posterior bulb (Fig. 6C).

The absence of homogeneous glomeruli in the adult AC3-/mice for all four OSN populations we examined suggests that AC3 is required for the formation of mature homogeneous glo- 

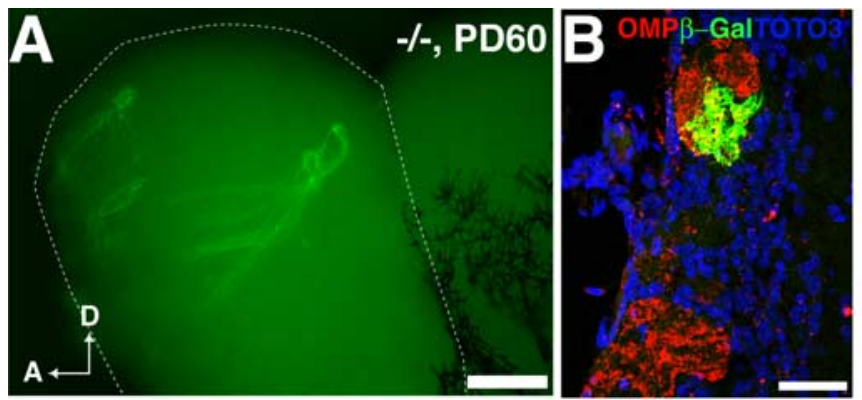

Figure 6. Deletion of $A C 3$ disrupts M72 axonal projections. $A$, Medial view of M72 axonal projections in whole-mount bulb of M72-GFP mice. M72 axons project to a novel anterior region, as well as the posterior dorsal bulb of a PD60 AC3 - / mouse. A, Anterior; D, dorsal. $\boldsymbol{B}, \boldsymbol{C}$, Persistence of heterogeneous M72 glomeruli in either the anterior $(\boldsymbol{B})$ or the posterior bulb $(\boldsymbol{C})$ in a 1-year-old AC3 - / - mouse. Red, 0MP; green, $\beta$-gal; blue, nucleic staining with T0T03. Scale bars: $\boldsymbol{A}, 0.5 \mathrm{~mm}$; (in $B$ ) $B, C, 50 \mu \mathrm{m}$.
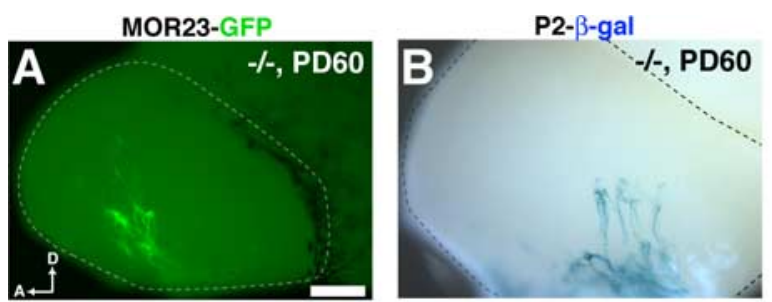

M71->P2- $\beta$-gal

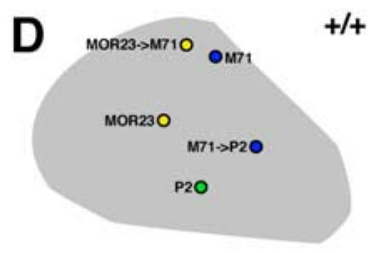

MOR23-GFP X MOR23->M71- $\beta$-gal
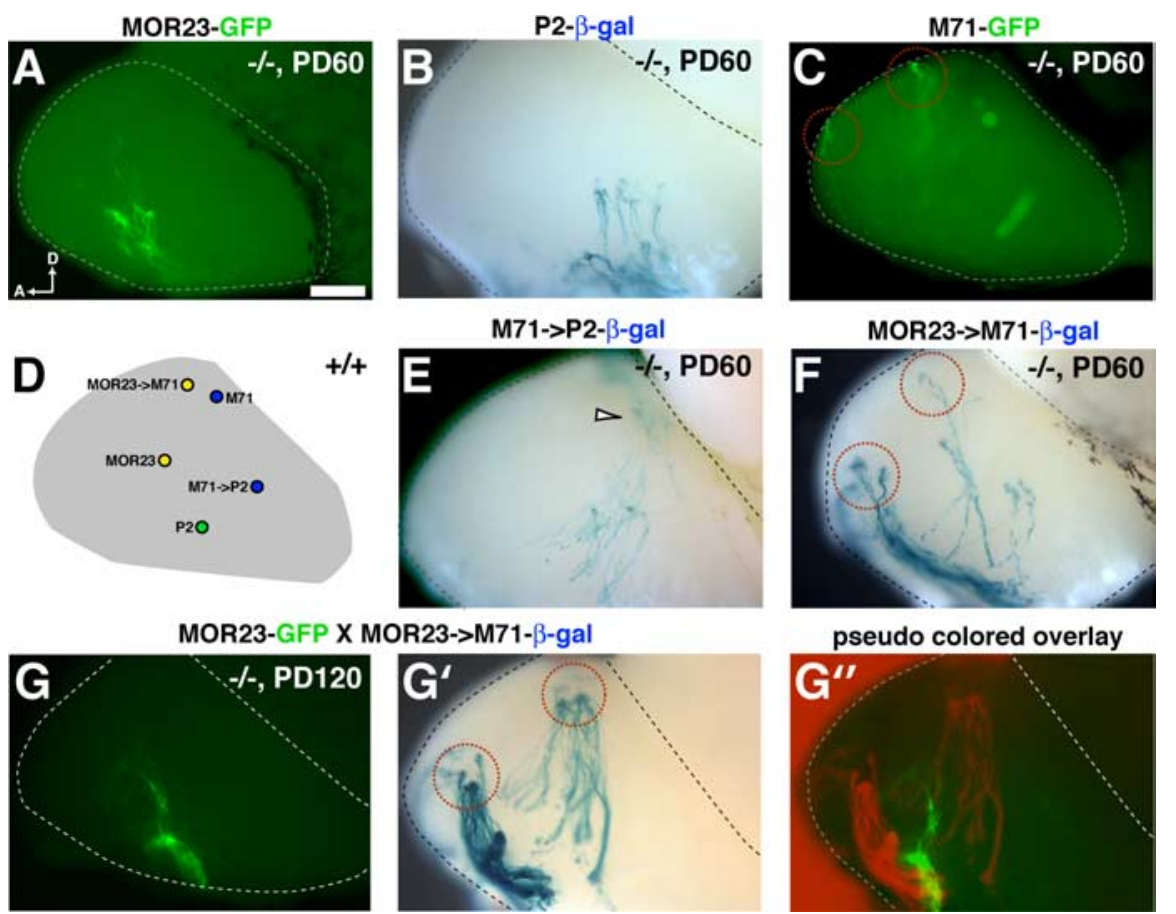

pseudo colored overlay

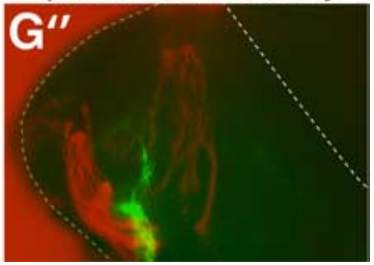

Figure 7. Determinants for the gross projection patterns of OSN axons in the AC3-/- background. $\boldsymbol{A}-\boldsymbol{C}$, Medial views of distinct projection patterns of axons from 0SNs expressing M0R23 ( $\boldsymbol{A}, \mathrm{GFP}), \mathrm{P} 2(\boldsymbol{B}, \beta \mathrm{gal})$, or M71 (C, GFP) in AC3 - / - mice at PD60. A, Anterior; D, dorsal. Red circles in Chighlight the segregation of M71 axonal projections in the anterior and posterior dorsal bulb. $D$, Schematic illustration of positions for various glomeruli in the bulb of $A C 3+/+$ mice. $E$, Medial view of projection pattern of $\mathrm{M} 71 \rightarrow \mathrm{P} 2$ axons in the AC3 - / - background at PD60. Arrow points to passing by axons innervating the accessory olfactory bulb (Levai et al., 2006), regardless of $A C 3$ genotype. $\boldsymbol{F}$, Projection pattern of MOR23 $\rightarrow$ M71 axons in the AC3 $-/-$ background at PD60. Red circles highlight the segregation of M0R23 $\rightarrow$ M71 axonal projections in the anterior and posterior dorsal bulb. G, $\mathbf{G}^{\prime}$, Medial views of projection patterns of M0R23 (G, GFP) and M0R23 $\rightarrow$ M71 ( $\left.\boldsymbol{G}^{\prime}, \beta \mathrm{gal}\right)$ axons in the same bulb of an AC3-/mouse at PD120. Red circles in $\mathbf{G}^{\prime}$ highlight the segregation of MOR23 $\rightarrow$ M71 axonal projections in the anterior and posterior dorsal bulb. G", Projections of M0R23 (green) and M0R23 $\rightarrow$ M71 (pseudocolored in red) axons are largely segregated in the aligned and superimposed image. Scale bar: (in $\boldsymbol{A}) \boldsymbol{A}-\boldsymbol{C}, \boldsymbol{E}-\boldsymbol{G}^{\prime \prime}, 0.5 \mathrm{~mm}$.

meruli. However, we noticed that M72 axons were sometimes highly compartmentalized within a glomerulus (Fig. 6B). Similar compartmentalization was also observed in older mice for MOR23 axons (Fig. 4I-K). Although a homogeneous glomerulus did not form, the capability of recognizing like axons was not completely lost in the absence of AC3. The aggregation of like axons within a glomerulus in AC3-/-mice suggests that the sorting of OSN axons uses factors in addition to AC3.

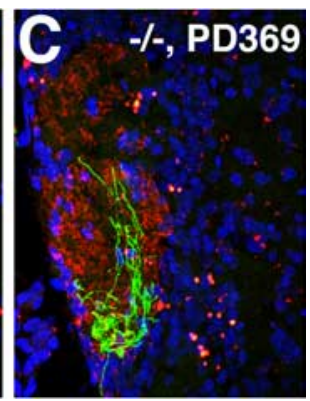

MOR23->M71- $\beta$-gal

Determinants for axonal projection patterns in the AC3-/- background The unusual projection patterns unmasked in the AC3-/- background (Fig. $7 A-C$ ) suggested that multiple factors act in concert with the receptor to promote proper glomerular formation. One possibility is that the events that differentially impact the selection of an OR locus for expression may also differentially affect the activation of factors involved in axonal projections. The unexpected anterior axonal projection patterns of the M71expressing OSNs in AC3-/- mice afforded us an opportunity to investigate this possibility further. We took advantage of two lines of receptor replacement mice involving M71 (Feinstein et al., 2004). In these mice, the coding region of one receptor is replaced with that of another receptor. Axons from OSNs expressing the substituted receptor normally converge into glomeruli located in novel positions (Fig. 7D).

In $\mathrm{M} 71 \rightarrow \mathrm{P} 2$ OR replacement mice, the $\mathrm{P} 2$ coding region is replaced by the M71 coding region together with IREStaulacZ; thus, M71 is expressed from the P2 locus together with the marker $\beta$-gal. In the AC3-/- background, we found that the $\mathrm{M} 71 \rightarrow \mathrm{P} 2$ axons showed a phenotype similar to that observed for endogenous P2 axons in AC3-/- mice; that is, few fibers could be found that project to the ventral medial bulb (Fig. $7 E)(n=3$ animals, ranging from PD10 to PD60). We did not observe projections of $\mathrm{M} 71 \rightarrow \mathrm{P} 2$ axons in the anterior bulb, the atypical projection region for axons of endogenous M71-expressing OSNs in the AC3-/- background (Fig. 7C). Thus, when AC3 was absent, the projection pattern in the receptor replacement condition correlated with that predicted by the locus rather than by the receptor.

We also examined the MOR23 $\rightarrow$ M71 receptor replacement mice. In the AC3-1- background, MOR23 axons projected only to the anterior bulb (Fig. 7A) whereas M71 axons projected to both the anterior and posterior bulb (Fig. $7 C)$. In the MOR23 $\rightarrow$ M71 replacement strains of an AC3-/- background, MOR23 $\rightarrow$ M71 axons were found in clusters in both the anterior and posterior dorsal bulb (Fig. $\left.7 F, G^{\prime}\right)(n=3$ animals, ranging from PD20 to PD120). Thus, again the projection pattern appeared to correlate with the locus rather than the receptor when AC3 is absent. Also of interest, we observed that the projections of endogenous MOR23 axons (Fig. 7G, GFP) and MOR23 $\rightarrow$ M71 axons (Fig. $7 G^{\prime}, \beta$-gal) did not appear to intermingle (Fig. $\left.7 G^{\prime \prime}\right)$ in the same bulb of an AC3-1- mouse. The segregation of these two populations of axons, both expressing the same receptor, further suggests that there are additional components that impact axonal identity. 


\section{Discussion}

A fundamental event in the establishment of proper olfactory projections is the formation of homogeneous glomeruli that are exclusively innervated by the same population of axons. Several critical steps underlying glomerular formation have recently begun to emerge. It had been originally demonstrated that the OR is required for the convergence of like axons (Mombaerts et al., 1996; Wang et al., 1998; Feinstein and Mombaerts, 2004; Feinstein et al., 2004). Previously, we and others have suggested that activation of the G-protein/cAMP signaling cascade is critically involved in OR-dependent axonal sorting (Imai et al., 2006; Chesler et al., 2007). Here, we show that the lack of AC3, a key component of the OR mediated cAMP dependent signaling cascade, perturbs the proper projections of individual OSN axonal populations and disrupts the formation of homogeneous glomeruli.

Under normal circumstances, multiple populations of axons initially coalesce heterogeneously. Subsequent activitydependent refinement ensures the exclusive homogeneous innervation of a given glomerulus by a single population of OSN axons, a process that is largely completed by PD60 (Zou et al., 2004) (D.-J. Zou, unpublished observation). The refinement process is retarded by sensory deprivation, resulting in the maintenance of heterogeneous glomeruli (Zou et al., 2004). The results presented here reveal that AC3 deficiency has even more pronounced effects on glomerular development than sensory deprivation by naris closure. In AC3-1- mice, homogeneous glomeruli for representative receptors were not observed at any age. Furthermore, the absence of AC3 resulted in aberrant anterior projections (Figs. 5, 6) and coinnervation (Chesler et al., 2007) of M71 and M72 axons. Neither of these outcomes has been observed in other manipulations of sensory activity.

The lack of AC3 may affect glomerular development in a number of ways. Although a weak AC3 signal has been reported in the mitral cells of adults (Visel et al., 2006), we favor a presynaptic role for its function. AC3 expression in mitral cells occurs after many glomeruli have already emerged. Moreover, it has been demonstrated previously that mitral cells are not required for OSN axonal convergence (Bulfone et al., 1998; St. John et al., 2003).

What then is the role of AC3 in OSNs beyond odorant detection? We can envision AC3 activity having several functions in OSNs, including the growth and steering of axons, the promotion of OSN survival, and the regulation of gene expression. The high levels of AC3 expression in OSN axons during the period of glomerular formation indicate that CAMP can be locally generated to steer the growth of axons (Song et al., 1997). Additionally, it has been reported that odorant evoked AC3 activation is required for the survival of OSNs after the removal of olfactory bulb in adults (Watt et al., 2004).

Elimination of AC3 clearly affects various OSN populations differently. Our results indicate a reduction in the number of P2-expressing cells. The lack of apparent P2 glomeruli in whole mount in the AC3-/- background (Fig. 3) may reflect the requirement of a minimal number of axons for glomerular formation and the interdependency of P2 axons (Ebrahimi and Chess, 2000). However, this explanation is not applicable to the absence of homogeneous MOR23 glomeruli in AC3-/- mice; despite relatively equal numbers of MOR23-expressing OSNs in AC3 $-/-$ and AC3 $+/+$ mice (Fig. 4E, F), homogeneous MOR23 glomeruli remain unformed in the AC3-/- background.

It remains to be determined whether AC3 function is also required to maintain the one receptor per neuron rule. It is possible that the absence of AC3 may promote OR gene switching (Shykind et al., 2004) or impede the elimination of OSNs expressing multiple receptors (Mombaerts, 2004). For example, both M71- and M72-expressing OSNs may be coexpressing receptors that normally project to the anterior dorsal bulb. However, we have thus far been unable to find preferential coexpression of receptors or coexpression of M71 and M72 (supplemental Fig. 1, available at www.jneurosci.org as supplemental material). In addition to any possible effects on OR feedback, cAMP signaling is a potent regulator of gene expression and, thus, may regulate genes important for glomerular formation (Imai et al., 2006).

Our results from receptor-replacement mice in an AC3-/background indicate that the variable projection patterns for individual axon populations associate with the activation of a given receptor locus rather than the specific receptor coding sequence alone (Fig. 7). Thus, the differences in gene expression profiles between individual OSN populations, regardless of the actual odorant receptor protein they express, are also likely important for determining their axonal projections. Variable gene profiles among different OSN populations are supported by the observations that molecules important for glomerular formation are differentially expressed among glomeruli (St. John et al., 2002; Cutforth et al., 2003; Serizawa et al., 2006). It has long been assumed that all OSNs are identical if they express the same receptor. This assumption has been recently challenged by the variable response profiles of individual MOR23 neurons (Grosmaitre et al., 2006). A difference in protein expression profiles among the OSNs expressing the same receptors could account for the atypical anterior projections of M71 and M72 axons in the AC3-/- background. However, direct evidence remains to be obtained to support this idea.

Our work suggests that an OR-mediated signaling cascade participates in local sorting of axons from OSNs that differ mainly by the receptor they express. In this model, the receptors function, at least in part, by stimulating AC3. The cAMP signals thus generated may directly influence growth cones and/or expression of guidance genes. Interestingly, cAMP activity has also been implicated in regulating the retinogeniculate projections in the visual system (Stellwagen and Shatz, 2002; Ravary et al., 2003; Nicol et al., 2007), as well as the organization of thalamocortical afferents in the somatosensory cortex (Welker et al., 1996; AbdelMajid et al., 1998). In these systems, cAMP activity appears to act, at least in part, by modulating spontaneous electrical activity (Stellwagen et al., 1999), or by changing the strength of synaptic transmission (Lu et al., 2003). The targets of cAMP and the processes it influences in the regulation of olfactory projections to the bulb remain to be identified.

\section{References}

Abdel-Majid RM, Leong WL, Schalkwyk LC, Smallman DS, Wong ST, Storm DR, Fine A, Dobson MJ, Guernsey DL, Neumann PE (1998) Loss of adenylyl cyclase I activity disrupts patterning of mouse somatosensory cortex. Nat Genet 19:289-291.

Bakalyar HA, Reed RR (1990) Identification of a specialized adenylyl cyclase that may mediate odorant detection. Science 250:1403-1406.

Baker H, Cummings DM, Munger SD, Margolis JW, Franzen L, Reed RR, Margolis FL (1999) Targeted deletion of a cyclic nucleotide-gated channel subunit (OCNC1): biochemical and morphological consequences in adult mice. J Neurosci 19:9313-9321.

Belluscio L, Gold GH, Nemes A, Axel R (1998) Mice deficient in G(olf) are anosmic. Neuron 20:69-81.

Buck L, Axel R (1991) A novel multigene family may encode odorant receptors: a molecular basis for odor recognition. Cell 65:175-187.

Bulfone A, Wang F, Hevner R, Anderson S, Cutforth T, Chen S, Meneses J, 
Pedersen R, Axel R, Rubenstein JL (1998) An olfactory sensory map develops in the absence of normal projection neurons or GABAergic interneurons. Neuron 21:1273-1282.

Chesler AT, Zou DJ, Le Pichon CE, Peterlin ZA, Matthews GA, Pei X, Miller MC, Firestein S (2007) A G protein/cAMP signal cascade is required for axonal convergence into olfactory glomeruli. Proc Natl Acad Sci USA 104:1039-1044.

Cutforth T, Moring L, Mendelsohn M, Nemes A, Shah NM, Kim MM, Frisen J, Axel R (2003) Axonal ephrin-As and odorant receptors: coordinate determination of the olfactory sensory map. Cell 114:311-322.

Ebrahimi FA, Chess A (2000) Olfactory neurons are interdependent in maintaining axonal projections. Curr Biol 10:219-222.

Feinstein P, Mombaerts P (2004) A contextual model for axonal sorting into glomeruli in the mouse olfactory system. Cell 117:817-831.

Feinstein P, Bozza T, Rodriguez I, Vassalli A, Mombaerts P (2004) Axon guidance of mouse olfactory sensory neurons by odorant receptors and the $\beta 2$ adrenergic receptor. Cell 117:833-846.

Grosmaitre X, Vassalli A, Mombaerts P, Shepherd GM, Ma M (2006) Odorant responses of olfactory sensory neurons expressing the odorant receptor MOR23: a patch clamp analysis in gene-targeted mice. Proc Natl Acad Sci USA 103:1970-1975.

Grubb MS, Thompson ID (2004) The influence of early experience on the development of sensory systems. Curr Opin Neurobiol 14:503-512.

Imai T, Suzuki M, Sakano H (2006) Odorant receptor-derived cAMP signals direct axonal targeting. Science 314:657-661.

Ishii T, Omura M, Mombaerts P (2004) Protocols for two- and three-color fluorescent RNA in situ hybridization of the main and accessory olfactory epithelia in mouse. J Neurocytol 33:657-669.

Katz LC, Shatz CJ (1996) Synaptic activity and the construction of cortical circuits. Science 274:1132-1138.

Kerr MA, Belluscio L (2006) Olfactory experience accelerates glomerular refinement in the mammalian olfactory bulb. Nat Neurosci 9:484-486.

Levai O, Feistel T, Breer H, Strotmann J (2006) Cells in the vomeronasal organ express odorant receptors but project to the accessory olfactory bulb. J Comp Neurol 498:476-490.

Lin DM, Wang F, Lowe G, Gold GH, Axel R, Ngai J, Brunet L (2000) Formation of precise connections in the olfactory bulb occurs in the absence of odorant-evoked neuronal activity. Neuron 26:69-80.

Lu HC, She WC, Plas DT, Neumann PE, Janz R, Crair MC (2003) Adenylyl cyclase I regulates AMPA receptor trafficking during mouse cortical "barrel" map development. Nat Neurosci 6:939-947.

Mombaerts P (2004) Odorant receptor gene choice in olfactory sensory neurons: the one receptor-one neuron hypothesis revisited. Curr Opin Neurobiol 14:31-36.

Mombaerts P, Wang F, Dulac C, Chao SK, Nemes A, Mendelsohn M, Edmondson J, Axel R (1996) Visualizing an olfactory sensory map. Cell 87:675-686.

Nicol X, Voyatzis S, Muzerelle A, Narboux-Neme N, Sudhof TC, Miles R, Gaspar P (2007) cAMP oscillations and retinal activity are permissive for ephrin signaling during the establishment of the retinotopic map. Nat Neurosci 10:340-347.

Ravary A, Muzerelle A, Herve D, Pascoli V, Ba-Charvet KN, Girault JA, Welker E, Gaspar P (2003) Adenylate cyclase 1 as a key actor in the refinement of retinal projection maps. J Neurosci 23:2228-2238.

Ressler KJ, Sullivan SL, Buck LB (1993) A zonal organization of odorant receptor gene expression in the olfactory epithelium. Cell 73:597-609.

Royal SJ, Key B (1999) Development of P2 olfactory glomeruli in P2internal ribosome entry site-tau-LacZ transgenic mice. J Neurosci 19:9856-9864.
Serizawa S, Miyamichi K, Takeuchi H, Yamagishi Y, Suzuki M, Sakano H (2006) A neuronal identity code for the odorant receptor-specific and activity-dependent axon sorting. Cell 127:1057-1069.

Shykind BM, Rohani SC, O'Donnell S, Nemes A, Mendelsohn M, Sun Y, Axel R, Barnea G (2004) Gene switching and the stability of odorant receptor gene choice. Cell 117:801-815.

Song HJ, Ming GL, Poo MM (1997) cAMP-induced switching in turning direction of nerve growth cones. Nature 388:275-279.

St. John JA, Pasquale EB, Key B (2002) EphA receptors and ephrin-A ligands exhibit highly regulated spatial and temporal expression patterns in the developing olfactory system. Brain Res Dev Brain Res 138:1-14.

St. John JA, Clarris HJ, McKeown S, Royal S, Key B (2003) Sorting and convergence of primary olfactory axons are independent of the olfactory bulb. J Comp Neurol 464:131-140.

Stellwagen D, Shatz CJ (2002) An instructive role for retinal waves in the development of retinogeniculate connectivity. Neuron 33:357-367.

Stellwagen D, Shatz CJ, Feller MB (1999) Dynamics of retinal waves are controlled by cyclic AMP. Neuron 24:673-685.

Treloar HB, Feinstein P, Mombaerts P, Greer CA (2002) Specificity of glomerular targeting by olfactory sensory axons. J Neurosci 22:2469-2477.

Trinh K, Storm DR (2003) Vomeronasal organ detects odorants in absence of signaling through main olfactory epithelium. Nat Neurosci 6:519-525.

Vassalli A, Rothman A, Feinstein P, Zapotocky M, Mombaerts P (2002) Minigenes impart odorant receptor-specific axon guidance in the olfactory bulb. Neuron 35:681.

Vassar R, Ngai J, Axel R (1993) Spatial segregation of odorant receptor expression in the mammalian olfactory epithelium. Cell 74:309-318.

Visel A, Alvarez-Bolado G, Thaller C, Eichele G (2006) Comprehensive analysis of the expression patterns of the adenylate cyclase gene family in the developing and adult mouse brain. J Comp Neurol 496:684-697.

Wang F, Nemes A, Mendelsohn M, Axel R (1998) Odorant receptors govern the formation of a precise topographic map. Cell 93:47-60.

Watt WC, Sakano H, Lee ZY, Reusch JE, Trinh K, Storm DR (2004) Odorant stimulation enhances survival of olfactory sensory neurons via MAPK and CREB. Neuron 41:955-967.

Welker E, Armstrong-James M, Bronchti G, Ourednik W, GheorghitaBaechler F, Dubois R, Guernsey DL, Van der Loos H, Neumann PE (1996) Altered sensory processing in the somatosensory cortex of the mouse mutant barrelless. Science 271:1864-1867.

Wong ST, Trinh K, Hacker B, Chan GC, Lowe G, Gaggar A, Xia Z, Gold GH, Storm DR (2000) Disruption of the type III adenylyl cyclase gene leads to peripheral and behavioral anosmia in transgenic mice. Neuron 27:487-497.

Yoshihara Y, Kawasaki M, Tamada A, Fujita H, Hayashi H, Kagamiyama H, Mori K (1997) OCAM: a new member of the neural cell adhesion molecule family related to zone-to-zone projection of olfactory and vomeronasal axons. J Neurosci 17:5830-5842.

Yu CR, Power J, Barnea G, O’Donnell S, Brown HE, Osborne J, Axel R, Gogos JA (2004) Spontaneous neural activity is required for the establishment and maintenance of the olfactory sensory map. Neuron 42:553-566.

Zhang X, Firestein S (2002) The olfactory receptor gene superfamily of the mouse. Nat Neurosci 5:124-133.

Zheng C, Feinstein P, Bozza T, Rodriguez I, Mombaerts P (2000) Peripheral olfactory projections are differentially affected in mice deficient in a cyclic nucleotide-gated channel subunit. Neuron 26:81-91.

Zou D-J, Feinstein P, Rivers AL, Mathews GA, Kim A, Greer CA, Mombaerts P, Firestein S (2004) Postnatal refinement of peripheral olfactory projections. Science 304:1976-1979. 